
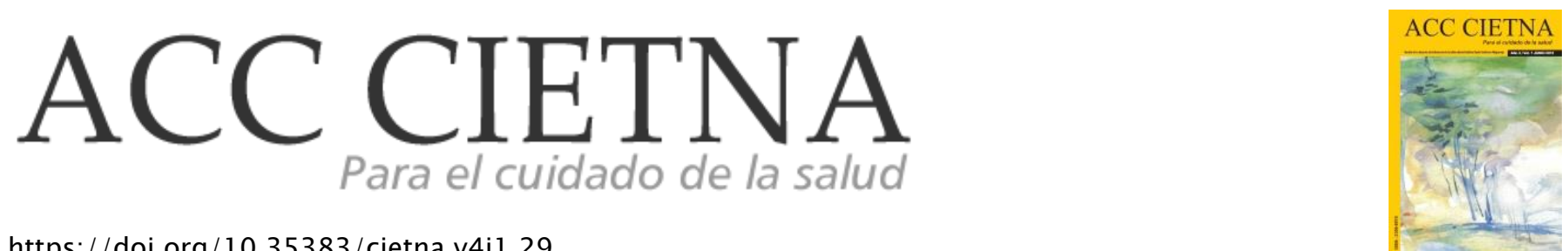

https://doi.org/10.35383/cietna.v4i1.29

\title{
Efectividad del modelo de la marea en la salud mental positiva de estudiantes de enfermería
}

\author{
Mendo Zelada Teresa ${ }^{1}$, Díaz Villena Noemí ${ }^{2}$, Castro de Marquina María ${ }^{3}$, Alfaro Ríos Segundo ${ }^{4}$ \\ , Vásquez Olcese Cesar ${ }^{5}$
}

\begin{tabular}{l}
\hline INFORMACIÓN DEL ARTÍCULO RESUMEN \\
\hline Historia del artículo:
\end{tabular}

Recibido el 23 de octubre de 2016

Aceptado el 15 de noviembre de 2016

\section{Palabras clave:}

Salud Mental Positiva

Modelo de la marea
La investigación se realizó en base a uno de los modelos y teorías intermedias de enfermería, el modelo de la marea, a fin de determinar su efectividad en la salud mental de las estudiantes de enfermería de la Facultad de Enfermería en una muestra de 65 estudiantes del VI ciclo, de diseño experimental de series de tiempo (antes-después), con grupo control y experimental a quienes se les aplicó la Escala de salud mental positiva de Lluch. En el grupo experimental se desarrolló el modelo de la marea, el que utilizó tres estrategias basadas en las prácticas narrativas y el enfoque centrado en soluciones: Árbol de la vida, Pregunta del milagro, Línea de tiempo. Los resultados muestran que antes de aplicar el modelo de la marea, el $14.3 \%$ y el $19.6 \%$ de los grupos experimental y control registran nivel alto de salud mental positiva y no se encuentra diferencia significativa en la puntuación media alcanzada; mientras que después de aplicar el modelo de la marea, el $50.0 \%$ de los integrantes del grupo experimental registró un nivel alto de salud frente al $25.0 \%$ del grupo control, se concluye que el modelo de la marea es efectivo al mejorar la salud mental positiva de las estudiantes.

\footnotetext{
${ }^{1}$ Licenciada en Enfermería. Licenciada en Psicóloga. Magister en Salud Pública. Docente Asociada T.C. Facultad de Enfermería, Universidad Nacional de Trujillo, Trujillo, Perú. Email: tmendo@unitru.edu.pe

${ }^{2}$ Licenciada en Enfermería. Magister en Enfermería mención Salud de la mujer y el niño. Docente Principal D.E. Facultad de Enfermería, Universidad Nacional de Trujillo, Trujillo, Perú.

${ }^{3}$ Licenciada Enfermería. Magister en Educación mención Psicología educativa. Docente Principal D.E. Facultad de Enfermería, Universidad Nacional de Trujillo, Perú.

${ }^{4}$ Médico pediatra. Magister en Medicina. Docente Principal D.E. Facultad de Medicina. Universidad Nacional de Trujillo, Trujillo, Perú. Email: salfaro@unitru.edu.pe

${ }^{5}$ Psicólogo. Magister en Docencia Universitaria. Docente T.P. Facultad Ciencias de la Salud. Universidad Privada del Norte, Lima, Perú. Email: cesar.vasquez@psicologos.com
} 


\section{Effectiveness of the model of the tide in positive mental health of nursing students}

\section{ABSTRACT}

Keywords:

Positive Mental Health

Model tide
The research was conducted on the basis of the models and intermediate nursing theories, the model of the tide, in order to determine their effectiveness in mental health nursing students at the School of Nursing in a sample of 65 students VI cycle, experimental design time series (before and after), with control and experimental group who were administered the scale Lluch positive mental health. Tree of Life, Miracle Question, Timeline: In the experimental model of the tide, which used three strategies based on narrative practices and solution-focused approach was developed. The results show that before applying the model of the tide, $14.3 \%$ and $19.6 \%$ of the experimental and control groups recorded high levels of positive mental health and is not significant difference in the average score achieved; while after applying the model of the tide, $50.0 \%$ of the members of the experimental group showed a high level of health from $25.0 \%$ in the control group, it is concluded that the model of the tide is effective in improving positive mental health of students.

\section{Introducción}

En las décadas recientes se han logrado muchos avances en el campo de la salud y en la atención del ser humano. La prolongación de la esperanza de vida, la modernidad y la globalización promueven la necesidad de las personas de encontrar, en las nuevas circunstancias que les rodean, los elementos para asumir los cambios biopsicosociales que les permitan encontrar un rol social que coincida con su identidad, organizar su comportamiento para darle sentido a su relación con el entorno y sostener un proyecto de vida el que, en muchos casos se ve obstaculizado por las necesidades insatisfechas a causa de la pobreza y otros aspectos 1 .

Esta dificultad puede explicar el surgimiento de problemas sociales que afectan a la población en general y repercuten en cuestiones públicas y comunitarias tales como la salud, el trabajo, la seguridad y la educación; de modo que no basta analizar la conducta aislada de la persona en todas las etapas de la vida sino que también debe tomarse en cuenta su interacción con el contexto social en que vive ${ }^{2}$.

A nivel mundial se reporta 450 millones de personas que se encuentran afectadas en su salud mental y, casi un millón de personas se suicidan cada año en todo el mundo. Los primeros resultados de las encuestas de salud mental realizadas en varios países de América Latina demuestran que cerca de $80 \%$ de las personas con problemas de salud mental no tienen acceso a los servicios de salud. En el Perú las personas de estratos medios y bajos serían las más afectadas. En Lima, del total de la población que reconoce haber padecido problemas de salud mental, sólo el $24,5 \%$ accedió a los servicios de salud, situación que es similar en la provincia de Trujillo, departamento de La Libertad $3,4,5$.

La salud física y la salud mental no se deben diferenciar porque cuando hay una enfermedad física, las capacidades psicológicas y las formas de relación se ven alteradas. Muchos problemas 
psicológicos se manifiestan con frecuencia a través de síntomas somáticos (insomnio, cefalea, taquicardia, etc.). Los adultos jóvenes no están exentos de esta problemática, ya que la situación actual en que viven genera múltiples roles y responsabilidades (estudio, trabajo, hogar, entre otros) y que muchas veces no saben enfrentarlos ${ }^{5}$.

La salud mental puede considerarse como un constructo que no tiene una definición por sí misma, por lo que, para darle contenido, es necesario recurrir a conceptos más concretos como el de "bienestar/malestar psíquico" "felicidad" o "satisfacción", que son, igualmente, abstractos. La salud mental es un concepto amplio que tiene que ver con el bienestar psicológico como parte del bienestar global; alude a temas como la satisfacción con la calidad de vida, el mantener una adecuada moral y autoestima ${ }^{5}$, no debe considerarse como algo independiente de la salud general; implica una concepción global e integral de la persona, que contempla los aspectos físicos, psicológicos y sociales de forma interrelacionada 6 .

A pesar de las diferentes perspectivas, el término salud mental es el concepto oficialmente aceptado para referirse tanto a la salud como a la enfermedad mental $y$, en la práctica las intervenciones van prioritariamente encaminadas a la recuperación de la salud, dando así un cierto sentido al macro concepto de salud mental. En la actualidad se hace necesario superar los conceptos de salud mental focalizados en la prevención o el tratamiento y avanzar hacia una intervención centrada en potenciar el desarrollo personal positivo.

La salud mental positiva es el estado de bienestar, de funcionamiento óptimo de la persona, que implica la promoción de las cualidades del ser humano facilitando su máximo desarrollo potencial que está determinado por la presencia de los siguientes criterios: satisfacción personal, actitud pro social, autocontrol, autonomía, resolución de problemas y auto actualización y, habilidades de relación interpersonal 7 .

Trabajar en salud mental positiva demanda el desarrollo de abordajes actualizados coherentes con el desarrollo personal dejando de lado la centralización en el problema tal como lo propone el modelo de la marea, desarrollado por Phil Barker desde $1974^{8}$, quien destaca la importancia central de desarrollar el conocimiento de las necesidades de la persona a través de un trabajo en colaboración, desarrollar una relación terapéutica a través de métodos diferenciados de delegación de poder activo. El centro de este esfuerzo es ayudar a las personas a utilizar sus voces como instrumento fundamental para definir su recuperación del malestar psíquico.

Este modelo identifica los conceptos necesarios para conocer las necesidades humanas de personas con problemas vitales y explica sistemáticamente fenómenos específicos e indica la naturaleza de las relaciones en una visión particular del mundo, emplea metáforas universales culturalmente significativas asociadas al poder del agua para representar los aspectos conocidos del distrés humano. El agua es "la metáfora central de la experiencia vivida de la persona y el sistema de cuidados que automodela la persona con la ayuda de la enfermera"9.

El modelo de la marea, es una visión específica del mundo, ayuda a la enfermera a empezar a comprender qué es la salud mental para una persona en concreto y se basa en diez principios, 
denominados "los diez compromisos" que constituyen la brújula metafórica para el profesional.

Estos principios son: i) Valorar la voz. Es la historia de la persona, abarca no sólo el relato del malestar sino también la esperanza de su resolución. ii) Respetar el lenguaje. Es animar a la persona a hablar en sus propias palabras, en su voz característica; iii) Desarrollar la curiosidad genuina para comprender mejor a la persona y la significación humana de la historia no desplegada de su vida; iv) Llegar a ser el aprendiz. La persona es el experto mundial en la historia de su vida. El profesional aprende qué es necesario hacer, más que dirigir; v) Utilizar el equipo de herramientas disponible: la historia de la persona contiene numerosos ejemplos de lo que ha funcionado o puede funcionar en ella y puede utilizar para desbloquear o crear la historia de la recuperación; vi) Trabajar un paso más allá. Se trabaja conjuntamente con la persona para crear una apreciación de lo que tiene que hacerse ahora; vii) Dar el don del tiempo. Encontrar el tiempo para "hacer lo que debe hacerse"; viii) Revelar la sabiduría personal que se utiliza para respaldar a la persona y guiar el viaje a la recuperación; ix) Saber que el cambio es constante en el viaje de la recuperación; x) Ser transparente, para ganar la confianza de la persona y ayudarla a comprender qué se está haciendo y por qué9.

Este modelo, proporciona a la enfermera un elemento educativo en el centro de la intervención interdisciplinaria, la búsqueda en la resolución de problemas y la promoción de la salud mental a través de métodos narrativos ${ }^{8}$.

En nuestro medio, no se han encontrado investigaciones con las variables de este estudio; sin embargo, Navarro ${ }^{10}$, en una investigación con estudiantes universitarios en Colombia describió las características de la salud mental positiva encontrando que los estudiantes presentan indicadores de salud mental positiva, con fuerte presencia de los factores de autocontrol y habilidades de relaciones personales, los demás factores se logran evidenciar aunque con menor presencia y se encuentran porcentajes considerablemente bajos para cada uno de los factores.

Por lo anteriormente mencionado, y siendo tan poco accesibles los servicios de salud mental, se hace necesario acercar a la población estrategias que permitan promover y recuperar la salud mental; en este sentido, se propone el presente estudio sustentado en las bases conceptuales de la salud mental positiva y en el modelo de la marea para la recuperación de la salud mental.

La combinación de algunos factores, como: el exceso de trabajo, la falta de tiempo o la incompatibilidad entre las relaciones personales y las obligaciones formativas, crea una alta prevalencia de malestar psicológico dentro de la población universitaria ${ }^{11}$; de allí, que es importante desarrollar programas de atención eficaces, encaminados a la prevención y/o recuperación de la salud mental de la futura cuidadora de salud. De esta manera, se contribuirá a un mejor rendimiento del estudiante que opta por una carrera de la salud así como se logrará contribuir a nuevas rutas de investigación.

Con el presente estudio, se trata de asistir a las estudiantes de enfermería y de conocer formas de crecimiento y desarrollo como personas, explorando cómo actualmente viven con y pueden 
ir más allá de sus problemas/necesidades para vivir y hacer así "enfermería en salud mental". La pregunta de investigación fue, ¿Es efectivo el modelo de la marea en la salud mental positiva de las estudiantes de enfermería de la Universidad Nacional de Trujillo, 2014? La hipótesis planteada: El modelo de la marea es efectivo en la salud mental positiva si los puntajes se incrementan y los objetivos fueron: Identificar el nivel de salud mental positiva en los factores: satisfacción personal, actitud pro social, autocontrol, autonomía, resolución de problema y auto actualización, habilidades de relación interpersonal de las estudiantes de enfermería antes de aplicar el modelo de la marea en la Universidad Nacional de Trujillo, 2014; Determinar el nivel de salud mental positiva en los factores: satisfacción personal, actitud pro social, autocontrol, autonomía, resolución de problema y auto actualización, habilidades de relación interpersonal de las estudiantes de enfermería al término de aplicación del modelo de la marea en la Universidad Nacional de Trujillo, 2014 y establecer la efectividad del modelo de la marea en la salud mental positiva de las estudiantes de enfermería de la Universidad Nacional de Trujillo, 2014.

\section{Metodología}

El estudio se realizó en estudiantes de la Facultad de Enfermería de la Universidad Nacional Trujillo matriculados en el VI ciclo, año académico 2014, de enfoque cuantitativo, de diseño experimental de series de tiempo (antes-después), con grupo control y experimental ${ }^{12}$.

La población, estuvo conformada por el total de estudiantes matriculados en el VI ciclo durante el periodo académico 2014 II en la Escuela académico profesional de Enfermería de la Facultad de Enfermería de la Universidad Nacional de Trujillo.
Para el tamaño de la muestra se utilizó el muestreo por conveniencia, siendo los integrantes de la muestra los estudiantes que cumpliendo con los requisitos de la población objeto de estudio aceptaron participar en la investigación. Se conformaron dos grupos, el grupo experimental con 14 estudiantes y el grupo control con 51 estudiantes $^{13}$. La unidad de análisis fue la estudiante matriculada en el VI ciclo de la Escuela Académico Profesional de Enfermería de la Facultad de Enfermería de la Universidad Nacional de Trujillo año académico 2014-II.

Los instrumentos usados fueron, la Escala para evaluar la salud mental positiva7, cuestionario conformado por 39 ítems positivos y negativos que saturan los distintos factores que componen el modelo de la salud mental positiva. Cada ítem del instrumento tiene cuatro opciones de respuesta: siempre o casi siempre, con bastante frecuencia, algunas veces y nunca o casi nunca. Los ítems positivos tienen puntajes máximos de 4 para siempre y de 1 para nunca o casi nunca. En Los ítems negativos la calificación fue inversa. Para la obtención de los niveles en cada una de los factores se realizó una media y desviación estándar. La confiabilidad de cada uno de los factores propuestos es de valores superiores a 0.419 . La confiabilidad del instrumento es en un índice de 0.893 coeficiente de Alfa de Cronbach. La correlación entre escala global y factores es estadísticamente significativa.

La correlación entre factores es estadísticamente significativa7. También se trabajó con el Modelo de la marea, para el cual se diseñó tres sesiones pragmáticas basadas en el enfoque de las prácticas narrativas y de la terapia centrada en soluciones, en los cuales se sustenta el modelo de la marea y se traduce en los principios: valorar la voz, respetar el lenguaje, desarrollar la curiosidad genuina, llegar a ser el aprendiz, revelar la sabiduría personal, ser transparente, utilizar el equipo de herramientas disponible, trabajar un 
paso más allá, dar el don del tiempo y, saber que el cambio es constante9.

En la sesión 1, se trabajó el "Árbol de la vida". Corresponde a un tipo de práctica narrativa comunitaria; esta sesión se desarrolló en tres momentos: 1) El dibujo y construcción individual del árbol, 2) La construcción colectiva del bosque, 3) Certificados y celebración. Los dos primeros momentos son para construir una historia alternativa, la que contiene las habilidades, talentos y sueños de cada estudiante y las historias detrás de estas y, la última etapa tiene como propósito asegurar que los participantes se vayan con el reconocimiento de los recursos, habilidades y vínculos con personas importantes en sus vidas. En la sesión 2, se trabajó con la Pregunta del milagro, técnica de proyección al futuro de la Terapia Centrada en las Soluciones. Se emplea para construir, con los estudiantes, lo que se denomina "objetivos útiles", que les permitirá guiar su trabajo y ayudar a identificar lo que ya van bien o sus avances. Se trata de "crear un futuro para una persona que se ha quedado congelada en el presente", así se propicia el cambio en su forma de comportarse, pensar o sentir.

La sesión 3 se trabajó la Línea de tiempo, estrategia que permite potenciar la "unidad" al mismo tiempo que potencia la diversidad, permitiendo a los participantes encontrar vínculos y trabajar juntos hacia un bienestar más amplio. Luego de las coordinaciones respectivas, el primer día de clases se aplicó la escala de Salud Mental Positiva (pre-test) a la totalidad de estudiantes y luego de calificarla se determinó la homogeneidad de la población, se formó por conveniencia el grupo experimental en el que se desarrolló las tres sesiones del modelo de la marea en jornadas de 4 horas cada día.
A los 45 días del término de la aplicación del modelo se administró la escala de Salud Mental Positiva (post-test) en los grupos experimental y control. Finalmente y respetando los principios éticos, se procedió a desarrollar el modelo de la marea en las estudiantes del grupo control que desearon participar.

Los puntajes obtenidos luego de la aplicación de la Escala de salud mental positiva a los estudiantes del grupo experimental y control antes y después de la aplicación del modelo, se ingresaron en la hoja de cálculo Excel y luego fueron exportados al paquete estadístico SPSS 22.0, que sirvió como soporte para el procesamiento de los datos.

Seguidamente se utilizó métodos y técnicas de: a) estadística descriptiva (distribución de frecuencias bidimensionales, numérica y porcentual de la variable e indicadores según grupo de estudio. Coeficiente de correlación, ítem-test corregido, para evaluar la validez de constructo del instrumento. Coeficiente de Confiabilidad Alfa de Cronbach, para evaluar la confiabilidad del instrumento. Medidas estadísticas de resumen: media aritmética y desviación estándar) y b) estadística inferencial (prueba de normalidad de Kolmogorov-Smirnov. Prueba paramétrica de $t$ de Student).La investigación se realizó teniendo en cuenta los principios de anonimato, confidencialidad, beneficencia y no maleficencia. 


\section{Resultados, análisis y discusión}

\section{Tabla 1}

Nivel de Salud Mental Positiva antes de la aplicación del modelo de la marea en estudiantes de enfermería integrantes del grupo experimental y control. Universidad Nacional de Trujillo, 2014.

\begin{tabular}{lcccc}
\hline Nivel & \multicolumn{3}{c}{ Grupo de estudio } \\
de \\
Salud \\
$\begin{array}{l}\text { Mental } \\
(+)\end{array}$ & & & & \\
\cline { 2 - 5 } & Experimental & Control \\
\hline Alto & 2 & 14,3 & 10 & 19,6 \\
Medio & 9 & 64,3 & 29 & 56,9 \\
Bajo & 3 & 21,4 & 12 & 23,5 \\
\hline Total & 14 & 100.0 & 51 & 100.0 \\
\hline
\end{tabular}

Fuente: Resultados de la aplicación de la Escala de Salud

Mental Positiva a estudiantes de la Facultad de Enfermería UNT
Tabla 2

Comparación de la Salud Mental Positiva de los estudiantes de enfermería integrantes del grupo experimental y control, antes de la aplicación del modelo de la marea. Universidad Nacional de Trujillo 2014.

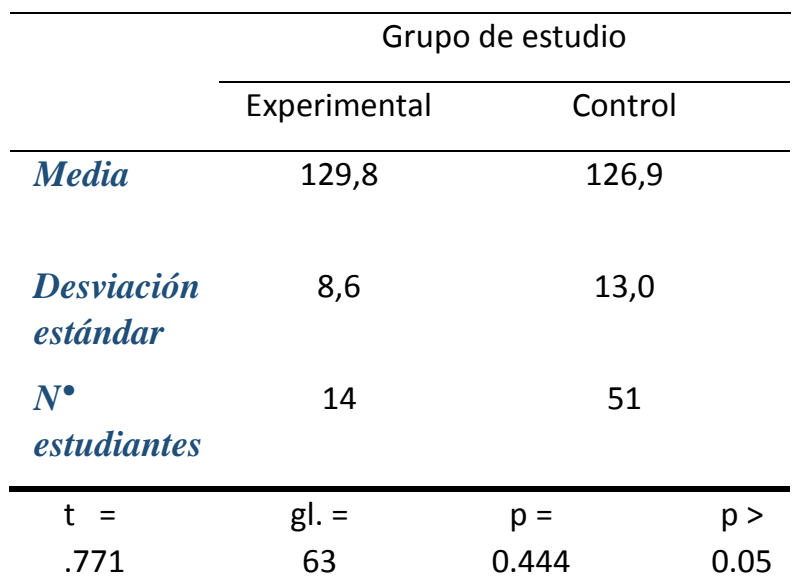


Tabla 3

Nivel de Salud Mental Positiva en estudiantes de enfermería integrantes del grupo experimental y control después de aplicar el modelo de la marea. Universidad Nacional de Trujillo, 2014.

\section{Grupo de estudio}

\begin{tabular}{lcccc}
\begin{tabular}{l} 
Nivel de \\
Salud \\
\cline { 2 - 5 } $\begin{array}{l}\text { mental } \\
(+)\end{array}$
\end{tabular} & Experimental & \multicolumn{2}{c}{ Control $^{+}$} \\
\hline Alto & 7 & 50,0 & 5 & 25,0 \\
Medio & 4 & 28,6 & 5 & 25,0 \\
Bajo & 3 & 21,4 & 10 & 50,0 \\
\hline Total & 14 & 100.0 & 20 & 100.0
\end{tabular}

Fuente: Resultados de la aplicación de la Escala de Salud Mental Positiva a estudiantes de la Facultad de enfermería - UNT

+: Después de la aplicación del modelo de la marea solo se pudo evaluar a 20 de las 51 integrantes del grupo control
Tabla 4

Comparación de la Salud Mental Positiva de los estudiantes de enfermería integrantes del grupo experimental y control, después de la aplicación del modelo de la marea. Universidad Nacional de Trujillo 2014.

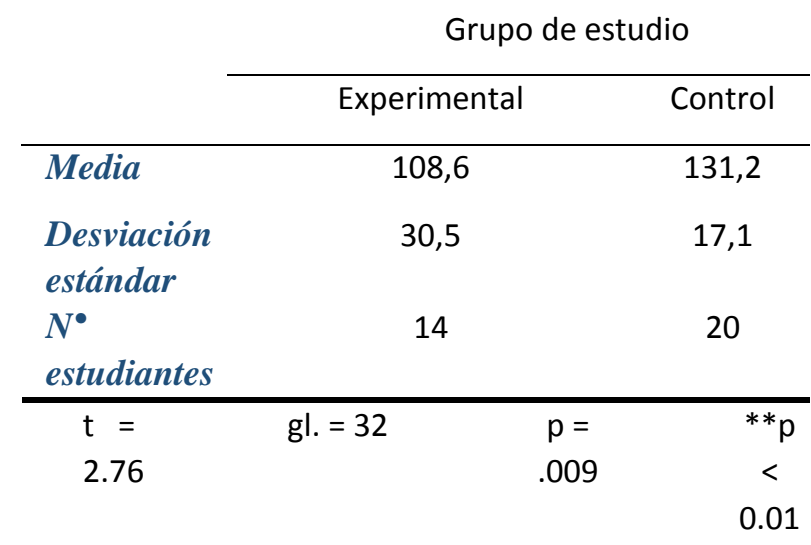

Antes de la aplicación del modelo de la marea, el mayor porcentaje de estudiantes de enfermería de los grupos experimental y control, presentaron una tendencia de medio y bajo en el nivel de salud mental positiva, así como en cada uno de los factores sin diferencia significativa entre ambos grupos, corroborándose la homogeneidad de los grupos de estudio, lo que evidencia la necesidad de un acompañamiento que promueva habilidades propias para generar un crecimiento personal. En 
la formación profesional de enfermeras, las estudiantes se enfrentan a problemas de adaptación a la vida universitaria y a las contradicciones entre la autonomía y los objetivos institucionales de la universidad con la demanda de eficacia y productividad de los servicios sumado a la realidad socioeconómica y geográfica en la que viven.

Salud mental positiva es la capacidad de percibir, de comprender y de interpretar las circunstancias que nos rodean para adaptarse a ellas y para cambiarlas en caso de necesidad ${ }^{14}$ y está determinado por la presencia de los factores satisfacción personal, actitud pro social, autocontrol, autonomía, resolución de problemas y auto actualización así como las habilidades de relación interpersonal?.

Estudiar la salud mental desde la perspectiva positiva, permite identificar habilidades personales y enfatizar en la promoción de recursos para un proceso constante de adaptación y equilibrio, que, si es favorable genera un movimiento progresivo de crecimiento personal y mantiene a la persona en el espacio de salud mental positiva ${ }^{15}$, siendo de mayor importancia en las personas que trabajan con personas como son los profesionales de enfermería. La sociedad cada vez más necesita profesionales de la salud capaces de brindar un cuidado humanizado desde su propia vivencia.

Estos resultados se asemejan a los reportados por Mendo ${ }^{16}$, en estudiantes del primer ciclo de la misma especialidad, semejanza que deja entrever que la persona tiene habilidades que le permiten adaptarse a diversas situaciones de cambio desde que ingresa a la universidad, sin embargo a pesar de las experiencias vividas en este tiempo de estudio, la salud mental positiva no mejora.
El modelo de la marea es un método para la recuperación de la salud mental, orientada a desarrollar el conocimiento de las necesidades de la persona a través de un trabajo en colaboración y una relación terapéutica mediante métodos diferenciados de delegación de poder activo8,9. Este modelo considera el valor inherente de la persona y el valor de relacionarse, la persona puede aprender de forma diferente su propio valor y el valor único de su existencia, es decir abre nuevas posibilidades para las personas ${ }^{8}$. Después del desarrollo del modelo de la marea se obtuvo que los porcentajes de niveles de salud mental positiva de medio a bajo, en el grupo experimental, se desplacen, al nivel alto de salud mental positiva, al igual que los puntajes promedio arrojando una diferencia significativa en relación al grupo control lo que demuestra la efectividad del modelo de la marea en la mejora del nivel de la salud mental positiva así como en sus factores, con excepción del factor satisfacción personal que no alcanza un valor de significancia pero sí muestra un incremento en la puntuación media.

No se han encontrado estudios que permitan contrastar los resultados; sin embargo, estudios guiados por el modelo de la marea indican su utilidad y precisión y ofrecen la seguridad de que la teoría es útil, práctica y accesible. Trabajar con el modelo de la marea permite articular la práctica y evidenciar habilidades invisibles de las personas ${ }^{8}$.

Los resultados de esta investigación, llevan a concluir que el modelo de la marea logró mejorar la salud mental positiva, con lo que se afirma que resultó efectivo en los estudiantes del sexto ciclo de enfermería que constituyeron el grupo experimental. Es así, que se hace necesario, que el docente universitario desarrolle habilidades para establecer una relación colaborativa que le permita 
al estudiante un crecimiento no sólo en el nivel académico; sino también, en lo personal. Por lo que, el modelo desarrollado en la presente investigación posibilita mejorar la salud mental positiva y contribuye a que el estudiante se desempeñe satisfactoriamente manteniendo su bienestar físico y mental. La calidad de la enseñanza universitaria exige un proceso de acompañamiento, consejería y tutoría al estudiante para que alcance su desarrollo integral, el que supone innovar estrategias que permitan la formación integral del futuro profesional.

\section{Conclusiones}

El nivel de salud mental positiva y los factores satisfacción personal, actitud pro social, autocontrol, autonomía, resolución de problema y auto actualización $y$, habilidades de relación interpersonal es muy homogéneo en los estudiantes de Enfermería de la Universidad Nacional de Trujillo, 2014 integrantes de los grupos experimental y control antes de aplicar el modelo de la marea.

El nivel de salud mental positiva mejora en el grupo experimental después de aplicar el modelo de la marea, con una diferencia altamente significativa en el factor resolución de problema y auto actualización; diferencia significativa en los factores actitud pro social, autocontrol, autonomía y habilidades de relación interpersonal y en el factor satisfacción personal sólo se incrementa la puntuación, sin diferencia significativa.

El modelo de la marea es efectivo en la salud mental positiva de las estudiantes de enfermería de la Universidad Nacional de Trujillo, 2014.

\section{Bibliografía}

1. MiNSA. Plan Nacional de Salud Mental. Lima: Comité Nacional de Salud Mental 2005-2006 del Consejo Nacional de Salud. Acceso 02 de diciembre 2013. Disponible en: http://www.minsa.gob.pe/dgsp/archivo/salu d_mental_documentos/01_Plan_Nacional_de_ Salud_Mental.pdf.

2. López E. Ministerio de Salud (MINSA) Situación de la Salud Mental en el Perú. 2007 Perú. Acceso 21 Setiembre 21 de 2007 Disponible en:

URL:http://www.minsa.gob.pe/portal/03Estra tegiasNacionales/10ESNSaludMental/esnsmmatinfo.asp

3. INSM. Estudio Epidemiológico Metropolitano en Salud Mental: Informe General. Lima: Anales de Salud Mental 18(1 y 2), 2002.

4. OPS. Salud mental en la comunidad. 2 da ed. Paltex, Washington, D.C. 2009

5. Caplan G. Aspectos preventivos en salud mental. Paidós, Barcelona, 1993.

6. Lluch M. Enfermería Psicosocial y de Salud mental: Marco conceptual y metodológico. Ediciones Universitat Barcelona. 2004.

7. Lluch M. Construcción de una escala para evaluar la salud mental positiva. Tesis doctoral. Universidad de Barcelona, 1999.

8. Brookes N. El modelo de la marea en la recuperación de la salud mental. En Raile, M. Marriner, T. Modelos y teorías en enfermería. 7ma edición. Barcelona, España: Elsevier. 2011

9. Cutcliffe J, McKenna H, Hyrkas K. Modelos de enfermería aplicación a la práctica. Editorial El Manual Moderno. México. 2011. 
10. Navarro B. Características de la salud mental positiva en estudiantes universitarios. Trabajo de investigación para optar el título de magíster en desarrollo social. Barranquilla, Colombia. 2008.

11. Tizón JL. Componentes psicológicos de la práctica médica. Una perspectiva desde la atención primaria. $4^{\mathrm{a}}$ edic. Biblaria. Barcelona, 1996

12. Hernández R, Fernández, C. Baptista P. Metodología de la Investigación. 5ta ed. Mc Graw-Hill. México, 2010.

13. Sánchez, H y Reyes C. Metodología y diseño De la investigación científica. Lima: Universidad Ricardo Palma. 2002

14. Lehtinen V. Action for Mental Health Activities co - funded from European Community Public Health Progrmames 1997-2004. Luxemburg: European Comunities and Stakes.2004. Disponible en URL: www.Ec.europa.eu/health/ph_determinants/li fe_styl e/mental/docs/action_9972004_en.pdf.
15. Fornés J \& Gómez J. Recurso y programas para la salud mental. Madrid: Enfo Ediciones. 2008.

16. Mendo T. Salud mental positiva en universitarios de primer y noveno ciclo, Trujillo. Tesis para optar el Título de Licenciada en Psicología. Universidad César Vallejo. Trujillo. 2014. 